\section{References}

1. Carrel T, Berdat P, Kipfer B, Eckstein F, Schmidli J. The reversed and bidirectional elephant trunk technique in the treatment of complex aortic aneurysms. J Thorac Cardiovasc Surg. 2001;122:587-91.

2. Heinemann MK, Buehner B, Jurmann MJ, Borst HG. Use of the "elephant trunk technique" in aortic surgery. Ann Thorac Surg. 1995;60:2-7.
3. Borst HG, Walterbusch G, Schaps D. Extensive aortic replacement using "elephant trunk" prosthesis. Thorac Cardiovasc Surg. 1983;31: $37-40$

4. Schepens MA, Dossche KM, Morshuis WJ, van den Barselaar PJ, Heijmen RH, Vermeulen FE. The elephant trunk technique: operative results in 100 consecutive patients. Eur J Cardiothorac Surg. 2002;21: $276-81$.

\title{
Acute intrathoracic gastric volvulus after laparoscopic fundoplication: Laparoscopic reduction and repair
}

\author{
Vishwanath Golash, MS, FRCS, Salalah, Sultanate of Oman
}

A

cute gastric volvulus is extremely rare after laparoscopic fundoplication. It is a true surgical emergency, and a delay in diagnosis can lead to fatal complications. Once the diagnosis is confirmed, nasogastric and gastroscopic decompression should be attempted. The surgical treatment involves reduction of the volvulus and prevention of recurrence. We present a patient who had an acute gastric volvulus after a Nissen fundoplication managed laparoscopically.

\section{Introduction}

Gastric volvulus can present acutely or chronically depending on the speed of onset of rotation and the extent of twist. It can also be classified on the basis of the axis of rotation. Organoaxial is the most common and is usually associated with a hiatus hernia. It classically presents with the clinical triad of retching, epigastric pain, and difficulty in passing a nasogastric tube.

\section{Clinical Summary}

A 23-year-old man presented with a 1-day history of increasingly severe upper abdominal and lower chest pain radiating to the back, retching, and abdominal distension. He felt nauseous but was unable to vomit. He had a past history of a laparoscopic Nissen fundoplication a year previously.

On admission, he looked unwell and was pale and tachycardic, with a temperature of $37.5^{\circ} \mathrm{C}$. He had upper abdominal distention and tenderness. His white cell count was 16.5/L, his hemoglobin level was $12 \mathrm{~g}$, and his $\mathrm{C}$-reactive protein concentration was

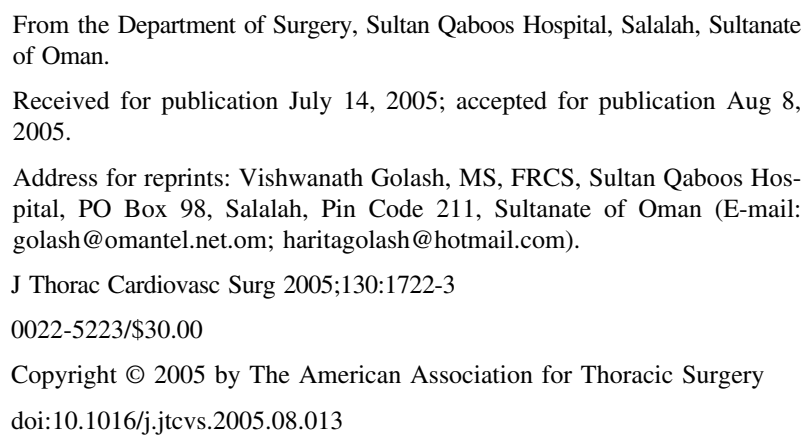

increased at $12 \mathrm{mg} / \mathrm{L}$. The results of the rest of his laboratory investigations, including the serum amylase and troponin measurements and electrocardiography, were normal.

The chest radiographs demonstrated 2 air-fluid levels within

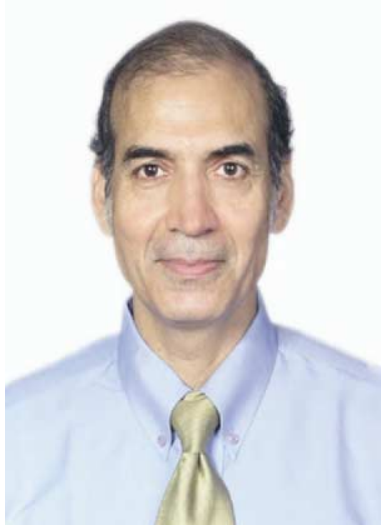

Dr Golash the chest at differing heights and a raised left hemidiaphragm. These findings were suggestive of a gastric volvulus. An urgent contrast-enhanced computed tomographic scan of his chest and abdomen showed 2 air-fluid levels in the posterior mediastinum (Figure 1). ${ }^{1}$ In lower cuts of the computed tomographic scan, a transition line was seen between the 2 air-fluid levels representing the site of the volvulus. In addition, in the lower cuts the left crus was not seen, suggesting the possibility of a rupture of the left crus.

An emergency gastroscopy revealed a large blood clot within the stomach, although no definite site of bleeding could be identified. The mucosa was inflamed at the previous site of fundoplication. The pyloric end of the stomach could not be reached. The stomach was decompressed at the end of the procedure, and it was believed that the bleeding was possibly caused by mucosal ischemia. A barium study confirmed the volvulus of the intrathoracic herniated part of the stomach. Barium reached the pyloric end of the stomach after 2 hours. He was taken for emergency surgical intervention that afternoon.

The diagnosis was confirmed at laparoscopy. A mark at the site of constriction was visible as a ring on the greater curvature of the stomach, suggesting that partial reduction of the volvulus had occurred and possibly after gastroscopy. Along with the stomach, the caudate lobe of the liver had also migrated into the chest. The herniated part of the stomach and liver was reduced into the abdomen. ${ }^{2}$ After adequate adhesiolysis in and around the hiatus and the posterior mediastinum, the esophagus was skeletonized, and an adequate intra-abdominal length of esophagus was freed. The hernial sac in the posterior mediastinum housing the volvulus was completely excised. The previous cruroplasty had torn apart with severe anatomic disruption. The hiatus was about $8 \mathrm{~cm}$ in diameter, and it was not possible to 


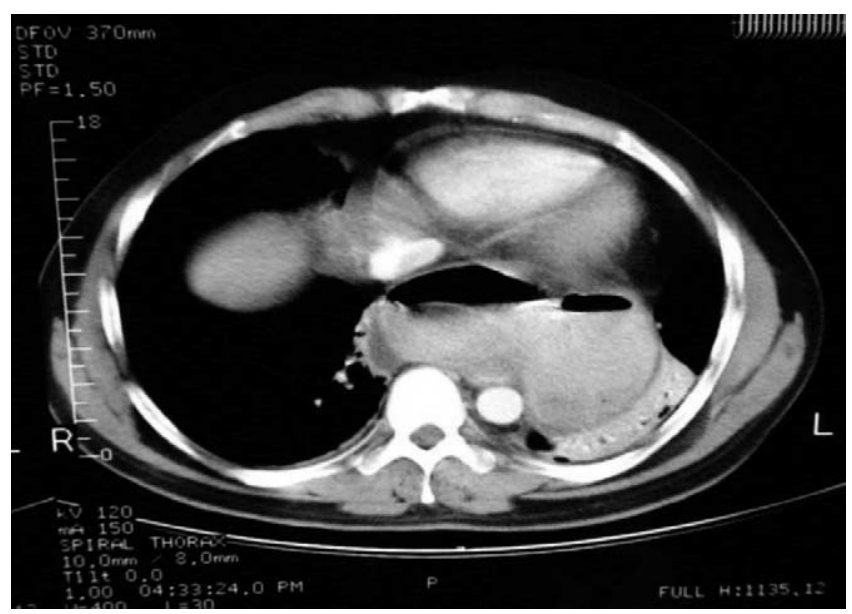

Figure 1. Chest computed tomogram showing 2 air-fluid level in the posterior mediastinum.

bring together the torn crurae. The hiatus was therefore repaired with a circular $10 \times 10 \mathrm{~cm}$ polypropylene mesh after making a radial slit with a $4-\mathrm{cm}$ keyhole for the esophagus (Figure 2). The mesh was sutured with interrupted nonabsorbable sutures around the hiatus, and the 2 free ends of the mesh were sutured together. ${ }^{3,4}$ A Nissen refundoplication was performed to prevent further reflux, and an anterior gastropexy was performed between the gastric fundus and diaphragm to reduce the chances of recurrence of gastric volvulus or herniation.

\section{Results}

The patient's recovery was uneventful. Outpatient endoscopy done 6 months after the operation showed no recurrence of the volvulus.

\section{Discussion}

Acute gastric volvulus is a rare condition and is difficult to diagnose, and only very few cases have been reported after laparoscopic fundoplication. The diagnosis is based on a high index of clinical suspicion and on radiologic investigations. A gastric volvulus can occasionally spontaneously reduce, and the diagnosis

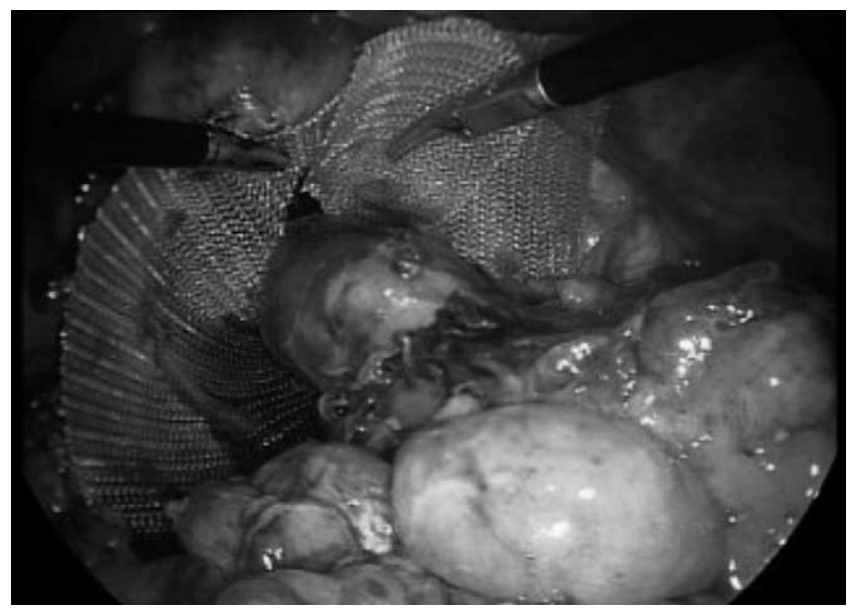

Figure 2. Circular mesh with radial slit.

might be missed in intermittent torsion. Surgical strategies include reduction of the volvulus, excision of the hernial sac, hiatal repair, fundoplication, and anterior gastropexy. ${ }^{5}$

\section{Conclusion}

A laparoscopic approach is safe and feasible in the repair of an acute intrathoracic gastric volvulus.

\section{References}

1. Cherukupalli C, Khaneja S, Bankulla P. CT Diagnosis of acute gastric volvulus. Dig Surg. 2003;20:497-9.

2. Pierre AF, Luketich JD, Fernando HC, Christie NA, Buenaventura PO, Litle VR, et al. Results of laparoscopic repair of giant paraoesophageal hernias: 200 consecutive patients. Ann Thorac Surg. 2002;74:1909-16.

3. Carlson MA, Condon RE, Ludwig KA, Schulte WJ. Management of intrathoracic stomach with polypropylene mesh prosthesis reinforced transabdominal hiatus hernia repair. J Am Coll Surg. 1998;187: 227-30.

4. Munteanu R, Copaescu C, Iosifescu R, Timisescu L, Dragomirescu C. Laparoscopic approach in large hiatus hernia-particular considerations. Chirurgia (Bucur). 2003;98:209-18.

5. Naim HJ, Smith R, Gorecki PJ. Emergent laparoscopic reduction of acute gastric volvulus with anterior gastropexy. Surg Laparosc Endosc Percutan Tech. 2003;13:389-91. 